This is the author's final, peer-reviewed manuscript as accepted for publication. The publisher-formatted version may be available through the publisher's web site or your institution's library.

\title{
Optimal intentional islanding to enhance the robustness of power grid networks
}

S. Pahwa, M. Youssef, P. Schumm, C. Scoglio, N. Schulz

\section{How to cite this manuscript}

If you make reference to this version of the manuscript, use the following information:

Pahwa, S., Youssef, M., Schumm, P., Scoglio, C., \& Schulz, N. (2013). Optimal intentional islanding to enhance the robustness of power grid networks. Retrieved from http://krex.ksu.edu

\section{Published Version Information}

Citation: Pahwa, S., Youssef, M., Schumm, P., Scoglio, C., \& Schulz, N. (2013). Optimal intentional islanding to enhance the robustness of power grid networks. Physica A: Statistical Mechanics and its Applications, 392(17), 3741-3754.

Copyright: @ 2013 Elsevier B.V.

Digital Object Identifier (DOI): doi:10.1016/j.physa.2013.03.029

Publisher's Link:

http://www.sciencedirect.com/science/article/pii/S037843711300246X

This item was retrieved from the K-State Research Exchange (K-REx), the institutional repository of Kansas State University. K-REx is available at http://krex.ksu.edu 


\title{
Optimal intentional islanding to enhance the robustness of power grid networks
}

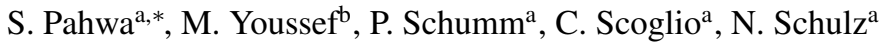 \\ ${ }^{a}$ Department of Electrical and Computer Engineering, Kansas State University, Manhattan, KS 66506-5204, USA \\ ${ }^{b}$ Network Dynamics and Simulation Science Laboratory, Virginia Bioinformatics Institute, Virginia Tech, Blacksburg, VA - 24061, USA
}

\begin{abstract}
Intentional islanding of a power system can be an emergency response for isolating failures that might propagate and lead to major disturbances. Some of the islanding techniques suggested previously do not consider the power flow model; others are designed to minimize load shedding only within the islands. Often these techniques are computationally expensive. We aim to find approaches to partition power grids into islands to minimize the load shedding not only in the region where the failures start, but also in the topological complement of the region. We propose a new constraint programming formulation for optimal islanding in power grid networks. This technique works efficiently for small networks but becomes expensive as size increases. To address the scalability problem, we propose two grid partitioning methods based on modularity, properly modified to take into account the power flow model. They are modifications of the Fast Greedy algorithm and the Bloom algorithm, and are polynomial in running time. We tested these methods on the available IEEE test systems. The Bloom type method is faster than the Fast Greedy type, and can potentially provide results in networks with thousands of nodes. Our methods provide solutions which retain at least $40-50 \%$ of the system load. Overall, our methods efficiently balance load shedding and scalability.
\end{abstract}

Keywords: Cascading, Mitigation, Intentional islanding, constraint programming, Fast Greedy, Bloom

\section{Introduction}

Power grids were designed for the purpose of transfer of electricity from the generators to the consumers, and were engineered keeping in mind the ever-increasing demand for electricity. However, in modern times the grid has reached a point where it has become very important to allow for its expansion in terms of technology and intelligence. Since the last few years, power grids have become increasingly interconnected. There are exchanges of large amounts of power over very long distances among different utilities to satisfy the increasing demand from the customers. The current setup is making the grid less stable and more vulnerable to intentional and unintentional failures. The system

\footnotetext{
* Corresponding author

Tel: +17855324690

Email addresses: sakship@ksu. edu (S. Pahwa), myoussef@vbi .vt.edu (M. Youssef), pbschumm@ksu . edu (P. Schumm), caterina@ksu.edu (C. Scoglio), noels@ksu.edu (N. Schulz)
} 
reliability and stability has been affected. As a matter of fact, there have been many occurrences of cascading failures in the recent past [1], [2], [3]. Defined by the North American Electric Reliability Corporation (NERC), a cascading failure is "the uncontrolled loss of any system facilities or load, whether because of thermal overload, voltage collapse, or loss of synchronism, except those occurring as a result of fault isolation" as mentioned in [4]. In simple words, when one failure leads to successive failure of other elements of the grid, leading to huge losses, the process is called a cascading failure. Some strategies such as reciprocal altruism [5], changing the dynamic equilibrium of the system to a point of self-organized criticality [6], [11], and different load shedding schemes [7], [8], [9], [10] have been suggested in the past for reducing the effects of cascading failures.

Intentional islanding of the power system is one such strategy. Intentional islanding can be defined as the intentional splitting of the grid into separate controllable parts or islands, each with its own independent generation. Intentional islanding may be accompanied by some load shedding in order to balance the generation and load in the sub-systems. Intentional islanding can be very helpful in isolating failures or localizing them within the region where they occurred and preventing them from spreading throughout the system. Several techniques have been proposed previously for islanding in power systems, such as those based on spectral analysis, slow coherency, ordered binary decision trees as well as optimization. An overview of these methods is described in Section 2.

In this paper, we propose a novel optimization formulation for optimal islanding of a power system. The formulation proposed here has been implemented using the CPLEX ILOG software by IBM [12], which transforms the logical constraints in the formulation to a mixed-integer program (MIP) internally. The formulation considers two parts of the system, the island (the region where the initial failure occurs) and the topological complement of the island. This technique aims at minimizing load shedding in both, the island and also its topological complement. Also, to limit the failure to a small portion of the system, the objective includes the minimization of the island size. However, the optimization problem is not scalable. The optimization takes a very long time to converge as the network size increases. This technique can be applied efficiently to small and medium sized systems such as the IEEE 14-node and 30-node networks [13] but it becomes computationally expensive for larger networks. For this reason, we propose two methods based on network partitioning and derived from the Fast Greedy algorithm [14] and the Bloom algorithm [15]. The original Fast Greedy and Bloom algorithms are based on the community detection metric, modularity [16], [17], [18], [19]. Since the concept of islanding is similar to that of detecting communities, by integrating the power flow model, we can make these algorithms realistic for islanding in power grids. Both the methods have a polynomial running time, but the Bloom approach is faster. In general, these methods are an efficient balance between the amount of load shedding and the algorithm scalability. Both have been tested on the IEEE 57-, 118- and 247-node networks, besides the 14-node and the 30-node networks. The 247-node network is a modification of the IEEE 300-node network and has been obtained as discussed in [7].

The island boundaries can be computed offline for all the three techniques and known to the operators in advance. Whenever a failure occurs, the predetermined set of transmission lines in the region where the island is needed, can be disconnected. Hence, the strategies can be implemented in real time. 
The main contributions of this paper are as follows:

- Proposing a novel optimization formulation for optimal islanding for minimizing load shedding in the island as well as the topological complement of the island.

- Proposing two polynomial time partitioning methods, based on modularity, and incorporating the power flow model to minimize load shedding in the islands and the island complement.

This paper is further organized as follows: Section 2 discusses the previous work that has been done in this field and the motivation for proposing the new techniques. The optimization formulation is discussed in detail in Section 3 and Appendix A. Section 4 discusses the two methods for islanding of the power grid. Numerical evaluation and comparison between the methods in terms of load shedding and the number of islands is described in Section 5 . Section 6 discusses the conclusions and scope for future work in this area.

\section{Related Work}

Since the past few years, the problem of intentional islanding is being studied as an important approach for isolating failures in the power grid [20], [21], [23], [25], [27], [29], [31]. It has been proposed by some researchers as an appropriate control action to protect the system when large disturbances take place. It is also considered to be an effective method to contain disturbances within a smaller area. Islanding leads to a faster restoration of the system to its initial state, as shown in [20], [21]. Different methods have been suggested to define islanding based on slow coherency generator grouping combined with graph theory, ordered binary decision diagrams (OBDD), linear and non-linear optimization as well as spectral methods. While slow coherency methods are among the first few methods proposed for islanding, spectral methods are fairly new.

The slow coherency methods are based on grouping the generators according to slow coherency and then trying to find the minimum cut-set from the interface network between the generator groups using some search techniques [20], [22], [23], [24], [25], [26]. The other category of methods deals with the ordered binary decision diagrams approach [27], [28]. For large-scale power networks, islanding using OBDD is an NP-hard problem. Hence, different two and three phase variants of this strategy have been suggested.

The authors of [29] and [30] present a mixed integer programming approach for optimal power grid islanding, both with the objective of minimizing load shedding only within the island. The authors of [29] also discuss connectivity constraints so that the nodes within each island are connected. As opposed to the techniques mentioned before, these two optimization techniques can form multiple islands at the same time.

The authors of [31] have used spectral matrix methods for islanding. They used successive bisection techniques based on the signs of the Laplacian eigenvalues to partition the grid. Cluster optimization using simulated annealing is suggested in [32]. In [33], separation of a power system into islands based on the second largest eigenvalue of the graph of the power system, also known as the algebraic connectivity, is presented. The authors have also shown that 
the computation time of this method has a linear relationship with number of transmission lines used for partitioning. However, these methods suffer from the drawback of the absence of a power flow model which makes them unrealistic for use within a power system.

While the above are the more common approaches for islanding in power grid, some researchers have also proposed the use of global search meta-heuristics such as genetic algorithm [21] and particle swarm optimization [34] for islanding as computationally efficient methods. A few of the above methods can form only two islands at a time, although the procedure can be repeated to obtain more islands.

In this work, we present an optimization formulation with logical constraints which are internally transformed to an MIP by the CPEX ILOG software, for islanding. The formulation has an improved objective function over the formulations already existing in literature. Our optimization formulation minimizes the load shedding both in the island itself as well as in the topological complement of the island, at the same time minimizing the size of the island to localize the failure in a small region around where it starts. We propose two network partitioning methods for larger systems for which the optimization is not scalable. We discuss these approaches in the following sections. We tested optimal islanding strategy on the IEEE 14-node and 30-node networks and it is a very effective method to create islands for these systems. The numerical evaluation of this method is shown in Section 5. However, optimal islanding is computationally expensive for larger systems. To overcome the problem of scalability, we propose two other methods for islanding.

\section{Optimal islanding for the power grids}

We present an optimization formulation to find the optimal islands when a failure takes place in any part of the grid. This optimization program has been solved on the CPLEX ILOG software by IBM. Binary variables are used in the formulation and the software uses the branch and cut method to solve the problem. It is an iterative process and continues until the optimal solution is found. The logical constraints in the constraint program are internally transformed by the software to a mixed-integer program.

Two different power flow models may be used for the analysis of a power grid, the full AC model or the linearized DC model. During emergency situations, quick and simple techniques are needed to control the unwanted situation. Even though slightly less accurate than the complete AC model, the DC power flow model serves the purpose in such situations. At the same time, the DC model is linear and does not face any convergence issues. Hence, for the purpose of intentional islanding, we have used the DC power flow model. A brief description of the two models is presented here.

\subsection{AC power flow model}

The $\mathrm{AC}$ power flow model comprises of the complete set of equations representing the non-linear dynamics of the power grid. Standard methods and algorithms such as Newton-Raphson method or Gauss-Seidel technique [35] are 
used to analyze the power grid using the AC power flow model. To find the power flowing through each link in the power grid, we first apply Kirchhoff's Current Law (KCL) at each node. We obtain a group of equations representing the relationship between the voltages and currents, which can be written in a matrix form as follows

$$
\left[\begin{array}{cccc}
\mathbf{Y}_{11} & \mathbf{Y}_{12} & \ldots & \mathbf{Y}_{1 N} \\
\mathbf{Y}_{21} & \mathbf{Y}_{22} & \ldots & \mathbf{Y}_{2 N} \\
\cdots & \ldots & \ldots & \cdots \\
\ldots & \ldots & \ldots & \cdots \\
\mathbf{Y}_{\mathrm{N} 1} & \ldots & \mathbf{Y}_{\mathrm{N}(\mathrm{N}-1)} & \mathbf{Y}_{\mathrm{NN}}
\end{array}\right]\left[\begin{array}{c}
\mathrm{V}_{1} \\
\mathbf{V}_{2} \\
\cdot \\
\cdot \\
\mathbf{V}_{\mathrm{N}}
\end{array}\right]=\left[\begin{array}{c}
\mathbf{I}_{1} \\
\mathbf{I}_{2} \\
\cdot \\
\cdot \\
\mathbf{I}_{\mathrm{N}}
\end{array}\right]
$$

Where $I_{k}$ is the current that enters the node from the generator/load side. The first matrix is called the admittance matrix or the $\mathbf{Y}_{\text {bus }}$ matrix. Each diagonal element $\mathbf{Y}_{\mathbf{k k}}$ equals the sum of the admittances of all branches connected to bus $k$. Every off-diagonal element $\mathbf{Y}_{\mathbf{j k}}$ where $j \neq k$ is the sum of admittances of all branches between bus $j$ and bus $k$ multiplied by -1 . Using Eq.(1), we obtain the following equation at node $k$

$$
\mathbf{V}_{\mathbf{1}} \mathbf{Y}_{\mathbf{k} \mathbf{1}}+\mathbf{V}_{\mathbf{2}} \mathbf{Y}_{\mathbf{k} \mathbf{2}}+\cdots+\mathbf{V}_{\mathbf{k}} \mathbf{Y}_{\mathbf{k k}}+\cdots+\mathbf{V}_{\mathbf{N}} \mathbf{Y}_{\mathbf{k N}}=\mathbf{I}_{\mathbf{k}}=\frac{P_{k}-j Q_{k}}{\mathbf{V}_{\mathbf{k}}^{*}}
$$

To find all unknown active power, reactive power, voltage magnitudes, voltage angles, different numerical techniques such as Gauss-Seidel or Newton-Raphson methods may be used.

The $\mathrm{AC}$ power flow model takes a longer time to converge and adds to the complexity of the islanding problem. Since the DC power flow model is linear, it does not add much to the complexity of the different islanding techniques we have discussed in this paper. Below we describe the fundamentals of the DC power flow model.

\subsection{DC power flow model}

The DC power flow model represents a linearization of the full AC model. In the AC model, let $V_{i}$ and $V_{j}$ represent the voltage at the buses $i$ and $j$, respectively. In addition, let $Y_{i j}$ represent the admittance of the transmission line between buses $i$ and $j$. the relation between real power, complex voltages and line impedance is expressed through the following equation which describes the amount of real power flowing through a transmission line

$$
P_{i j}=\left|V_{i}\right|\left|V_{j}\right|\left|Y_{i j}\right| \cos \left(\delta_{i}-\delta_{j}+\theta_{i j}\right)
$$

where $\theta_{i j}$ is the phasor angle of the admittance $Y_{i j}$. To obtain the DC power flow model, the following assumptions are applied to Eq.(3) as follows

- Voltage angle differences are small, i.e. $\sin \left(\delta_{i j}\right) \approx \delta_{i j}$.

- Flat Voltage profile: All voltage magnitudes are considered to be 1 p.u. 
- Line resistance is neglected since $R<<X$.

Applying Taylor expansion on Eq.(3) around the operating voltage, and neglect the coupling between the power flow and the voltage, we obtain

$$
P_{i j}=\frac{\delta_{i j}}{x_{i j}}
$$

where $\delta_{i j}$ is the difference in phase shift angle between the voltages at the sending and receiving buses, and $x_{i j}$ is the reactance of the transmission line. The DC power flow equation (4) can be written in matrix form where $P$ is the $N \times N$ matrix of power flows between each node $i$ and $j$ in the network, $\delta$ is the $N \times 1$ vector of phase angles and $X$ is the $N \times N$ weighted adjacency matrix, each element of which represents the reactance of a transmission line. It is a real number if a line is present between two nodes, and 0 otherwise. In matrix form,

$$
[P]=[b][\delta]
$$

The matrix $[b]$ represents the imaginary part of the $Y_{b u s}$ matrix of the power grid, where $b_{i j}=-\frac{1}{x_{i j}}$ and $b_{i i}=$ $\sum_{i \in N}-b_{i j}$ for $i \neq j$. We usually assume that there is a reference node with voltage angle equals 0 (which is the same node as the slack generator, in the most general sense). The power handled by each node is the net sum of all the ingoing and outgoing power flows at that node as follows:

$$
P_{i}=\sum_{j=1}^{N} P_{i j}=\sum_{j=1}^{N}\left(-b_{i j} \delta_{i j}\right)
$$

The total load at each node is given, while the phase angles are computed using the following equation:

$$
[\delta]=[b]^{-1}[P]
$$

\subsection{Optimal islanding}

In the optimization, we wish to contain the failure in a small part of the network and prevent it from spreading through the system causing a larger disturbance. Hence, the created island should be small in size, while the power delivered from the generators is maximized to satisfy the loads. The number of islands is represented by $n_{i s l}$, and we denote $k$ to be the island index such that $k=1 \ldots n_{i s l}$. The number of islands is given as an input to the formulation and is mainly limited by the number of generators present in the network. We need to make sure that every island has at least one generator so that it can sustain when separated from the rest of the network. As a result, the number of islands is always less than or equal to the number of generators in the network.

We denote the power grid topology to be $G$, the island topology to be $g_{k}$, the group of links that interconnects the island with its topological complement to be $l_{k}$, and the topological complement of the island to be $T_{k}=G \backslash\left\{l_{k} \cup g_{k}\right\}$. Moreover, we denote $s$ to be the index that distinguishes between the two partitions of the power grid due to the 
existence of an island $k$ i.e. for island $k, s=1$ denotes the island topology $\left(g_{k}\right)$ and $s=2$ denotes the island topological complement $\left(T_{k}\right)$. For a given power grid, islands are constructed such that the amount of generation/load change from normal operation and the island size are minimized as follows

$$
\begin{array}{r}
\text { Minimize } A \sum_{i=1}^{i=N} \sum_{k=1}^{k=n_{i s l}} \mid \text { power }_{i}-d_{i}^{k} \mid \\
+B \sum_{i=1}^{i=N} \sum_{j=1}^{j=N} \sum_{k=1}^{k=n_{i s l}} \mu_{i, j}^{k, 1}
\end{array}
$$

where $N$ is the number of nodes, $A$ and $B$ are optimization parameters. All the data inputs and variables are described in Table C.1.

The first part of the objective function aims at minimizing the load shedding in the island and the complement with power $_{i}$ being the original load of each node $i$ and $d_{i}^{k}$ being the new load after partitioning the grid, whose value is decided based on the formulation and the constraints. The smaller the difference between the original and the new values, the better is the result of islanding. The links which interconnect the different islands are chosen by the optimization such that the removal of those links does not cause additional damage to the network in terms of load loss. The second part of the objective function aims at minimizing the size of the island to limit the failure to a small region of the network. The variable $\mu$ counts the size of the island in terms of number of links. The subscript $i, j$ refers to the link $i-j$ and the superscipt $k, 1$ refers to the presence of the links within $(s=1)$ the island $k$. The values of parameters $A$ and $B$ can be used to decide the dominant part in the optimization. In this case, more weight is given to the first part, which is minimization of load shedding.

The islanding topology and power flow constraints are discussed in detail in the Appendix A. We tested optimal islanding strategy on the IEEE 14-node and 30-node systems and it is a very effective method to create islands for these systems. The numerical evaluation of this method is shown in Section 5. However, optimal islanding is computationally expensive for larger systems. The running time to solve the constraint programming problem for IEEE 14-node and 30-node networks is 45 minutes and 3.5 hours, respectively. To overcome this problem of scalability, we propose two methods based on modularity for islanding of power grids.

\section{Islanding based on modularity}

A community is usually a set of well connected nodes that are less connected to the remaining network. If we think of a power grid to be a network of nodes connected by transmission lines, we can use the power flow model incorporated into a community detection algorithm to "partition" the grid or to form islands such that this island formation leads to minimum load shedding in the system. Out of the numerous community detection algorithms, we select two among the faster and more efficient algorithms for island formation: Fast Greedy [14] and Bloom [15]. We 
modify these two algorithms by incorporating the power flow model and imposing some conditions to adapt them to a power grid.

In general, modularity is a quality function that determines the quality of the partitions on a scale of 0 to 1 , with 1 being the best value. For a general network, modularity can be defined as:

$$
Q(P, G)=\frac{1}{2 L} \sum_{i=1}^{N} \sum_{j=1}^{N}\left(a_{i j}-\frac{d_{i} d_{j}}{2 L}\right) \delta_{c_{i} c_{j}},
$$

where $N$ is the number of nodes and $L$ is the number of edges in the graph $G ; a_{i j}$ represents the existence of an edge between nodes $i$ and $j, d_{i}$ represents the degree of node $i$ or the number of nodes connected to node $i$. $P$ is the partition of the graph that divides it into communities by the quality measure $Q$. The communities of nodes $i$ and $j$ are represented as $c_{i}$ and $c_{j}$ respectively. The $\delta$-function is 1 if nodes $i$ and $j$ are in the same community $\left(c_{i}=c_{j}\right)$, otherwise it is 0 .

The objective of the two methods presented in this work is to minimize the amount of load shedding in the system (both island and complement) and is given as:

$$
J=\text { Minimize }_{i=1}^{N} \sum_{k=1}^{n_{i s l}} \mid \text { power }_{i}-d_{i}^{k} \mid
$$

This objective is the same as the first part of the objective function of the optimization, as shown in Equation 8 . However, we do not impose any restrictions on the size of the islands as described in the second part of Equation 8 because any such restriction would only reduce the quality of the islands and result in higher load shedding. Thus, the size of the islands is decided by the partitioning methods themselves. Similar to the optimization, the number of islands is limited by the number of generators in the network and the modified partitioning methods make sure that every island has at least one generator. Both methods are polynomial in complexity, but the Bloom method is faster than the Fast Greedy.

\subsection{Islanding using Fast Greedy type method}

The original Fast Greedy algorithm is an example of an agglomerative algorithm that begins with a number of partitions equal to the number of nodes in the network and merges these partitions depending on the benefit to the objective. For a power grid with $N$ nodes, we begin with $n_{i s l}^{\prime}=N$ islands and a $n_{i s l}^{\prime} \times n_{i s l}^{\prime}$ benefit matrix $\Delta J$ from Equation 10. Thus, at the beginning, each node is an individual island. According to the algorithm, the pairwise benefit $\Delta J_{r, s}=J_{\text {new }}-J_{\text {old }}$ is computed for every pair of islands $I s l_{r}$ and $I s l_{s} . J_{\text {new }}$ is the value of the objective after combination of islands and $J_{\text {old }}$ is the value of the objective in the current state. This benefit is computed for each potential pair of islands by a linear programming problem for minimal load shedding, which is basically the objective of the problem. Thus, the pair of islands that gives the largest decrease in the objective value or in other words, the minimal load shedding, is merged. This merged pair is now an individual island and can be merged with any other islands to continue the process. After every merge, the benefit matrix $\Delta J$ shrinks in size and must be 
recomputed. One of the requirements that this method must meet is the presence of at least one generator in every island. The required number of islands, $n_{i s l}<=n_{g e n}$, where $n_{\text {gen }}$ is the number of generators in the system, is given as an input. Once there are $n_{g e n}$ islands, if $n_{i s l}=n_{g e n}$, the process stops. However, if $n_{i s l}<n_{g e n}$, the process of "superislanding" begins by combining two or more of these $n_{\text {gen }}$ islands to reach the final goal of attaining $n_{i s l}$ islands after which the process stops. The process of superislanding also happens in the same way as above. The size of $\Delta J$ is the same as the final number of islands $n_{i s l}$ at the end of the process. The original Fast Greedy approach has a running time complexity of $O\left(N^{2} \log N\right)$ and the incorporated linear programming load shedding scheme adds a complexity of $O\left(N^{3}\right)$. Thus, this method is polynomial in complexity with a running time of $O\left(N^{5} \log N\right)$. The algorithm for this method is shown in Appendix B.

\subsection{Islanding using Bloom type method}

The Bloom type method begins with a few seed nodes and the islands grow from these seeds by adding adjacent nodes one at a time. In the original Bloom algorithm, the selection of the initial seeds is a stochastic process. However, in the case of islanding, we imposed all the generators in the network to be the initial seeds so that the number of islands at the beginning is equal to $n_{\text {gen }}$. This step is to make sure that every island has at least one generator. The nodes which are covered by the islands are said to be in the "covered" set and the other nodes are said to be in the "uncovered" set. Thus, at the beginning, all the generator nodes are a part of covered. We maintain a set of "boundary" nodes for each island. These boundary nodes have at least one connection to the island they are boundary nodes for, and they may become a part of that island. Again, the required number of islands, $n_{i s l}$ should be less than or equal to $n_{\text {gen }}$. All the islands are grown in parallel from the initial seeds, as opposed to growing one community at a time in the original Bloom. This is because the original Bloom has only one stochastically chosen seed node at a time whereas the modified Bloom for islanding has multiple seeds nodes determined right at the beginning. Similar to the Fast Greedy approach, this approach also computes a benefit matrix $\Delta J_{N \times n_{g e n}}$ after every merge which expresses the change to the objective value if any adjacent node merges into an island, using the linear programming problem for minimal load shedding. The node that adds the maximum benefit to the objective function (or the minimal load shedding) is chosen to merge with the island and moves from the boundary set to the covered set and its adjacent nodes move to the boundary set from the uncovered set. The nodes that already are a part of one island cannot be a part of any other island to avoid any overlapping of islands. The algorithm continues until all the nodes of the system are covered.

The original Bloom approach has an average running time of roughly $O(N)$ and a worst case running time of roughly $O\left(N^{2}\right)$. The linear programming contributes $O\left(N^{3}\right)$. Thus, the average running time of this approach is roughly $O\left(N^{4}\right)$ and the worst case running time is roughly $O\left(N^{5}\right)$. Bloom type approach is computationally more efficient than the Fast Greedy approach and in general, gives better results for load shedding. The algorithm for this method is described in Appendix C. 
Few different partitioning methods have been described in literature for partitioning of power grids as mentioned before. However, to the best of our knowledge, no other network partitioning algorithms using modularity have been applied previously for this purpose. The two approaches - Fast Greedy and Bloom, are very promising algorithms for network partitioning, in terms of complexity and are among the faster and highly efficient algorithms.

\section{Results}

Optimal islanding has been tested on the IEEE 14-node and the 30-node networks and the two modularity-based methods have additionally been tested on the 57-, 118- and 247-node networks [13]. Results and comparisons are shown through tables and graphs. The original 30-node network has only 2 generators, but to make it more suitable for islanding, 7 other generators were introduced in the system and the generation was equally divided among these 9 generators. Those nodes in the 30-node network that were carrying no load were converted into the 7 additional generator nodes. Similarly, the original 247-node system has 1 large generator and 7 other small distributed generators. The generation was equally divided among all these 8 generators and then island formation was carried out. These islanding techniques would be very suitable for the future grid which would have the incorporation of more distributed generation.

Table C.2 shows the average percentage of total load that remains after islanding in the 14-node network, using each of the three strategies. There are 2 generators in the system and hence, there are 2 islands. The number of islands is represented by $n_{i s l}$. The results show that the islands formed by each of the two modularity-based methods are exactly matching with the islands formed by the optimization. Also, as indicated in the table, the techniques can preserve around $86 \%$ of the load on average per island, in the system. The island structure is shown in Figure C.2. The circles represent the nodes and the connections between them are the transmission lines. The big circles represent the generator nodes and the small circles are the load nodes. Every island is represented by a separate color and nodes belonging to the same island have the same color.

Optimal islanding on the 30-node system, with 9 generators and 5 islands, is a more challenging case. Hence, we explain this case in detail. We apply the optimal islanding strategy on the IEEE 30-node system with 5 islands as the input. The topology of the islands and their topological complements are shown in Figure C.3. The dotted lines (- - ) are the transmission lines that interconnect every island with its topological complement. Sub-figures [a][e] represent individual islands connected to their topological complement and sub-figure [f] represents the complete 5-island structure. Every island is represented by a separate color and all nodes of the same color belong to an island. The gray colored nodes belong to the topological complement of the island in each sub-figure. The big circles represent the generators and the small circles represent the load nodes.

As shown in Figure C.3, every island has at least one generator and two transmission lines to guarantee the island connectivity. In addition, the test results show that due to line capacities, a few nodes experience load shedding but majority of the loads do not need any load shedding. Hence, the power generation is reduced at some generators. 
Another reason that a generator reduces its output power is that the total load in the island becomes less than its normal output generation. Table C.3 shows the results for the 30-node system with 9 generators and 5 islands. The optimization can converge up to at most 5 islands. The table indicates that the optimization performs better than the modularity-based methods in this case. Each merging decision made by these two methods is irreversible such that the local improvement at one step might not prove to be the best choice eventually and might lead to situations such as the one with the 30-node system where these methods give solutions which are below the optimal. Refinements to these methods might help to improve their performance to give results closer to optimal solution. Nevertheless, still approximately $60 \%$ of the load is maintained using these approaches which is a substantial gain when compared to the collapse of the entire system. Figure C.4 represents the different islanding structures for the optimal, Bloom type and, the Fast Greedy type approach respectively. This difference in the islanding structures is an indication of the difference in the amount of load shedding by the different methods.

In Tables C.4, C.5, C.6, we show the results for the 57-,118- and the 247-node systems for the two modularitybased methods. Since the optimization is not scalable for networks much larger than 30-nodes, further tests are done using only the Fast Greedy and Bloom approaches.

For the 57-node network, both the methods perform very well and preserve $95-96 \%$ of the load in the network. There are 2 generators in this network and so the tests were carried out for 2 islands. We did not add more generators to this network as in the earlier case of the 30-node network because we wanted to test the approaches on the original network, without altering the existing generation and load. In the previous case of the 30-node network, modifications were made to fully exploit the possibilities with the optimization problem and to see the limits to which it may be used.

For the 118-node system, we tested the two approaches for different number of islands from 2 to 6 . For each case, we see that the Bloom type approach performed better than the Fast Greedy type and the two approaches, in general, showed a good performance. We also see that the Bloom type method has the minimum load shedding with 3 islands whereas the Fast Greedy type has its best results with 6 islands. Figures C.5 and C.6 show the 6 island structures for the 118-node system with the Bloom and the Fast Greedy type approaches respectively. Again, different islands are represented by different colors and the big circles represent the generator nodes.

For the 247-node system, once again, in general, Bloom type method performs better than the Fast Greedy type. The best case for the Bloom type approach is the one with 2 islands in which it preserves about $68 \%$ of the system load. On the other hand, the Fast Greedy type performs the best with higher number of islands with about $59 \%$ system load preserved for 5 and 6 islands.

\section{Conclusions}

Intentional islanding in power systems has become an important subject of research because there is a need to find efficient solutions for the increasing frequency of blackouts. We explored three different schemes for intentional 
islanding in power grid - 1) a constraint optimization formulation for optimal islanding, 2) Bloom type and 3) Fast Greedy type approaches, based on modularity. The optimization formulation considers two parts of the system - the island and its topological complement - and works towards minimizing the load shedding in both parts of the system. It is capable of forming multiple islands and is a very efficient scheme for small networks, maintaining about 80$90 \%$ of the load after islanding. However, due to its exponential complexity, this method cannot be used for large networks.

We developed two approaches based on modularity, with the DC power flow model incorporated into them, for islanding in medium and large networks. The Bloom type and the Fast Greedy type methods were tested for the 57-, 118- and the 247-node networks, in addition to the 14- and the 30-node networks. These approaches also had the objective of minimizing load shedding, both in the island and the complement. They, in general, performed efficiently for all these systems, maintaining, at an average, at least $50 \%$ of the total load in the network.

It was imposed that every island must have at least one generator for independent survival. With more distributed generation, there would be better islanding opportunities and these methods will be well suited for the power grid of the future.

There is scope for future work in this area in the following directions:

- Using the AC power flow model for the power flow calculations for greater accuracy of results.

- Testing the islanding strategies under multiple failure scenarios.

- Simplifying the optimization formulation using semi-definite programming or other linearization techniques.

- Proposing refinements to the heuristics to bring the results closer to optimal solutions and to consider larger and more interconnected power systems as test cases.

\section{Acknowledgement}

The authors would like to thank the following funding sources: This work was partially supported by two Department of Energy grants, Kansas Wind Consortium DE-EE0000555, and DE-EE0003812 Resourceful Kansas. 


\section{Appendix A. Optimal islanding for the power grids - Constraints}

The optimization described previously has two sets of constraints: Topological constraints to ensure proper physical implementation of the islanding scenario, and power flow constraints describing the DC power flow model, which directs the flow of power through the power grid network. These constraints are given and described in detail below:

Appendix A.1. Topological constraints

$$
\begin{aligned}
& \sum_{k=1}^{k=n_{i s l}} \mu_{i, j}^{k, 1} \leq a_{i, j} \forall i, j=1 \ldots N \\
& \mu_{i, j}^{k, 1}+\mu_{i, j}^{k, 2} \leq 1 \forall i, j=1 \ldots N, k=1 \ldots n_{i s l} \\
& \sum_{i=1}^{i=N} \sum_{j=1}^{j=N} \mu_{i, j}^{k, s} \geq 4 \forall k=1 \ldots n_{i s l}, s=1,2 \\
& \sum_{j=1}^{j=N}\left(\mu_{i, j}^{k, 1}+\mu_{i, j}^{k, 2}\right) \geq 1 \forall i=1 \ldots N, k=1 \ldots n_{i s l} \\
& \sum_{k=1}^{k=n_{i s l}}\left(\mu_{i, j}^{k, 1}+\mu_{i, j}^{k, 2}\right) \leq a_{i, j} n_{i s l} \forall i, j=1 \ldots N \\
& \sum_{j=1}^{j=N} \sum_{k=1}^{k=n_{i s l}} \mu_{i, j}^{k, 1} \geq 1 \forall i=1 \ldots N \\
& \left(\sum_{j=1}^{j=N}\left(\mu_{i, j}^{k, 1}+\mu_{j, i}^{k, 1}\right) \geq 1\right) \\
& \Rightarrow\left(\sum_{j=1}^{j=N}\left(\mu_{i, j}^{k, 2}+\mu_{j, i}^{k, 2}\right)=0\right) \forall i=1 \ldots N, k=1 \ldots n_{i s l} \\
& \left(\sum_{j=1}^{j=N}\left(\mu_{i, j}^{k, 2}+\mu_{j, i}^{k, 2}\right) \geq 1\right) \\
& \Rightarrow\left(\sum_{j=1}^{j=N}\left(\mu_{i, j}^{k, 1}+\mu_{j, i}^{k, 1}\right)=0\right) \forall i=1 \ldots N, k=1 \ldots n_{i s l} \\
& \left(\sum_{j=1}^{j=N} \mu_{i, j}^{k, 1} \geq 1\right) \\
& \Rightarrow\left(\sum_{j=1}^{j=N} \sum_{k^{\prime}=1, k^{\prime} \neq k}^{k^{\prime}=n_{i s l}} \mu_{i, j}^{k^{\prime}, 1}=0\right) \forall i=1 \ldots N, k=1 \ldots n_{i s l}
\end{aligned}
$$




$$
\begin{gathered}
\mu_{i, j}^{k, s}=\mu_{j, i}^{k, s} \forall i, j=1 \ldots N, k=1 \ldots n_{i s l}, s=1,2 \\
\mu_{i, i}^{k, s}=0 \forall i=1 \ldots N, k=1 \ldots n_{i s l}, s=1,2
\end{gathered}
$$

The group of inequalities in A.1 - A.11 describes the topological constraints for creating the islands.

Inequality A. 1 imposes that the decision variable $\mu_{i, j}^{k, 1}$ can equal 1 only if the transmission line $(i, j)$ exists in the power grid i.e. $a_{i, j}=1$. In other words, if transmission line $(i, j)$ does not exist in the power grid $\left(a_{i, j}=0\right)$, the decision variable $\mu_{i, j}^{k, 1}$ equals 0 for all the islands $k=1 \ldots n_{i s l}$. Also this inequality guarantees that the transmission line $(i, j)$ can belong to at most one island $k, s=1$.

In inequality A.2, a transmission line $(i, j)$ can either be a part of the island $\mu_{i, j}^{k, s=1}=1$ or its complement $\mu_{i, j}^{k, s=2}=1$, or an interconnecting line between two islands. In the latter case, the transmission line does not belong to any island and the decision variables $\mu_{i, j}^{k, s=1}$ and $\mu_{i, j}^{k, s=2}$ equal 0 for all $k$. This ensures that the interconnecting transmission lines do not carry power flow.

Inequality A.3 ensures that the number of transmission lines in every island and its topological complement is at least 2. Notice that the transmission lines are undirected (even though the power flowing through the lines is directional). Hence the decision variables $\mu_{i, j}^{k, s}$ and $\mu_{j, i}^{k, s}$ are equal and the right-hand side of this inequality is 4.

Inequality A.4 ensures that every node $i$ in the power grid is assigned to either an island or to its topological complement and there is at least one transmission line that connects node $i$ with another node that belongs to the same island component. In addition, in inequality A.5, every line $(i, j)$ can belong to an island or its topological complement, and it can belong to any combination of them at most $n_{i s l}$ times.

Inequality A.6 guarantees that every node $i$ is assigned to an island. In other words, for every node $i$, there is at least one transmission line $(i, j)$ that connects node $i$ with another node $j$ in island $k, s=1$. Notice that inequalities A.6 and A.5 together ensure that each node belongs to an island.

Constraints A.7, A.8 and A.9 are formulated using the logical constraints. Each constraint has two parts, the conditional constraint (left-hand side), and the actual constraint (right-hand side). If the conditional constraint is true, the actual constraint is applied to the problem. As shown in constraint A.7, if there is at least a transmission line that is connected to node $i$ in island $k, s=1$ imposing that node $i$ belongs to island $k, s=1$, it implies that there is no transmission line that is connected to node $i$ in the topological complement $k, s=2$ imposing that node $i$ does not belong to the topological complement $k, s=2$. Constraint A.8 shows the case for the topological complement in the conditional constraint such that if there is at least one transmission line that is connected to node $i$ in the topological complement $k, s=2$, it implies that node $i$ does not belong to island $k, s=1$. Constraint A.9 ensures that if node $i$ has at least one transmission line in island $k, s=1$, node $i$ does not have any link in other islands $k^{\prime} \neq k$. This constraint implies that node $i$ can only belong to one island $k, s=1$ and it does not belong to other islands.

Inequality A.10 says that the line is symmetric or $i-j$ is the same as $j-i$, and inequality A.11 avoids self connecting 
nodes or self-loops.

\section{Appendix A.2. Power flow model constraints}

All the power flow calculations are based on the DC power flow model [35]. The DC model is linear and it does not add significantly to the complexity of the optimization formulation. The use of the DC model allows the optimization to easily converge for a few test cases in spite of its complexity. The DC model has been used in the recent works [29], [30] for islanding using MIP.

$$
\begin{gathered}
\delta_{\text {generator }(1)}^{k}=0 \forall k=1 \ldots n_{\text {isl }} \\
\left(\mu_{i, j}^{k, 1}+\mu_{i, j}^{k, 2}=1\right) \\
\Rightarrow\left(\left|b_{i, j}\left(\delta_{i}^{k}-\delta_{j}^{k}\right)\right| \leq c_{i, j}\right) \forall i, j=1 \ldots N, k=1 \ldots n_{\text {isl }} \\
d_{\text {gen }(g)}^{k} \leq 0 \forall g=1 \ldots n_{\text {gen }}, k=1 \ldots n_{\text {isl }} \\
d_{\text {gen }(g)}^{k} \geq 1.05 * \operatorname{power}_{\text {gen }(g)} \forall g=1 \ldots n_{\text {gen }}, k=1 \ldots n_{\text {isl }} \\
d_{\text {load }(l)}^{k} \leq \operatorname{power}_{\text {load }(l)} \forall l=1 \ldots n_{\text {loads }}, k=1 \ldots n_{\text {isl }} \\
d_{\text {load }(l)}^{k} \geq \alpha_{\text {power }} \\
\text { load }(l) \\
\forall l=1 \ldots n_{\text {loads }}, k=1 \ldots n_{\text {isl }}
\end{gathered}
$$

The group of constraints A.12 - A.17 represents the DC power flow model equations, the upper bounds, and the lower bounds of the generated power and loads. For DC power flow model, we assume that node 1 is the slack generator with voltage angle equals 0 as shown in equation A.12. The logical constraint A.13 indicates that whether a line $(i, j)$ belongs to an island or to the complement, the amount of power it can carry is always restricted by a finite capacity of the transmission line, given by $c_{i, j}$. The second part of this logical constraint is the main equation of the DC power flow model which computes the amount of power flowing through a link. Inequalities A.14 and A.15 represent the bounds on the amount of generated power from each generator $\operatorname{gen}(g)$ for each island scheme $k$. Thus, the generation is made flexible so that any fluctuations in the load can be accounted for. The inequalities A.16 and A.17 represent the bounds on the delivered loads. We impose the lower bound on the delivered power at each node to be a fraction $\alpha$ of the total load, where $\alpha$ is a real number between 0 and 1 . Thus, we allow load shedding in the network but at the same time a minimum fraction $\alpha$ of the load must be satisfied in each node.

In addition to the topological constraints and power flow model constraints, we use the network flow model [36] to ensure that every island is a single component in which all buses are interconnected by transmission lines. 


\section{Appendix B. Algorithm for Fast Greedy type approach for intentional islanding}

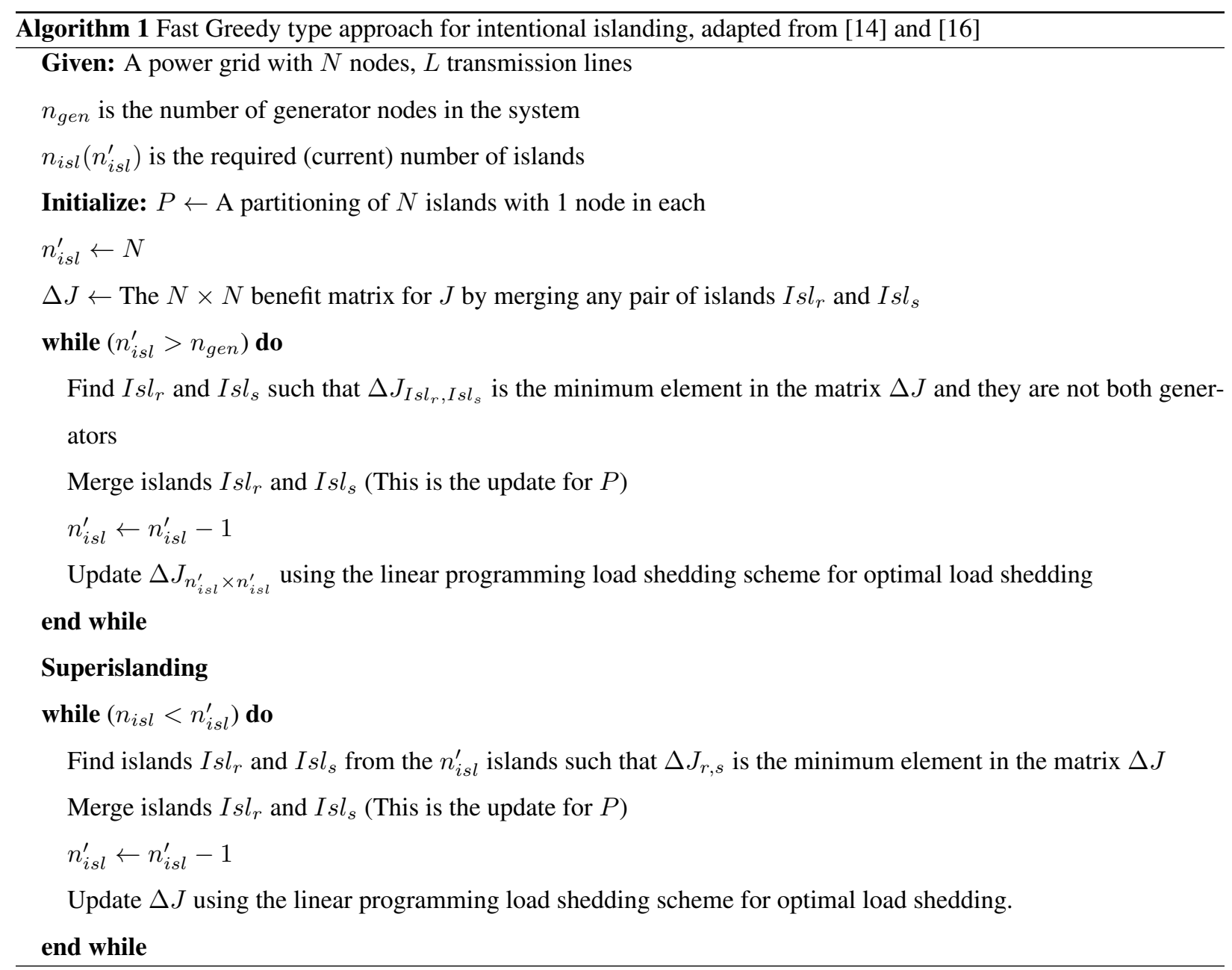




\section{Appendix C. Algorithm for Bloom type approach for intentional islanding}

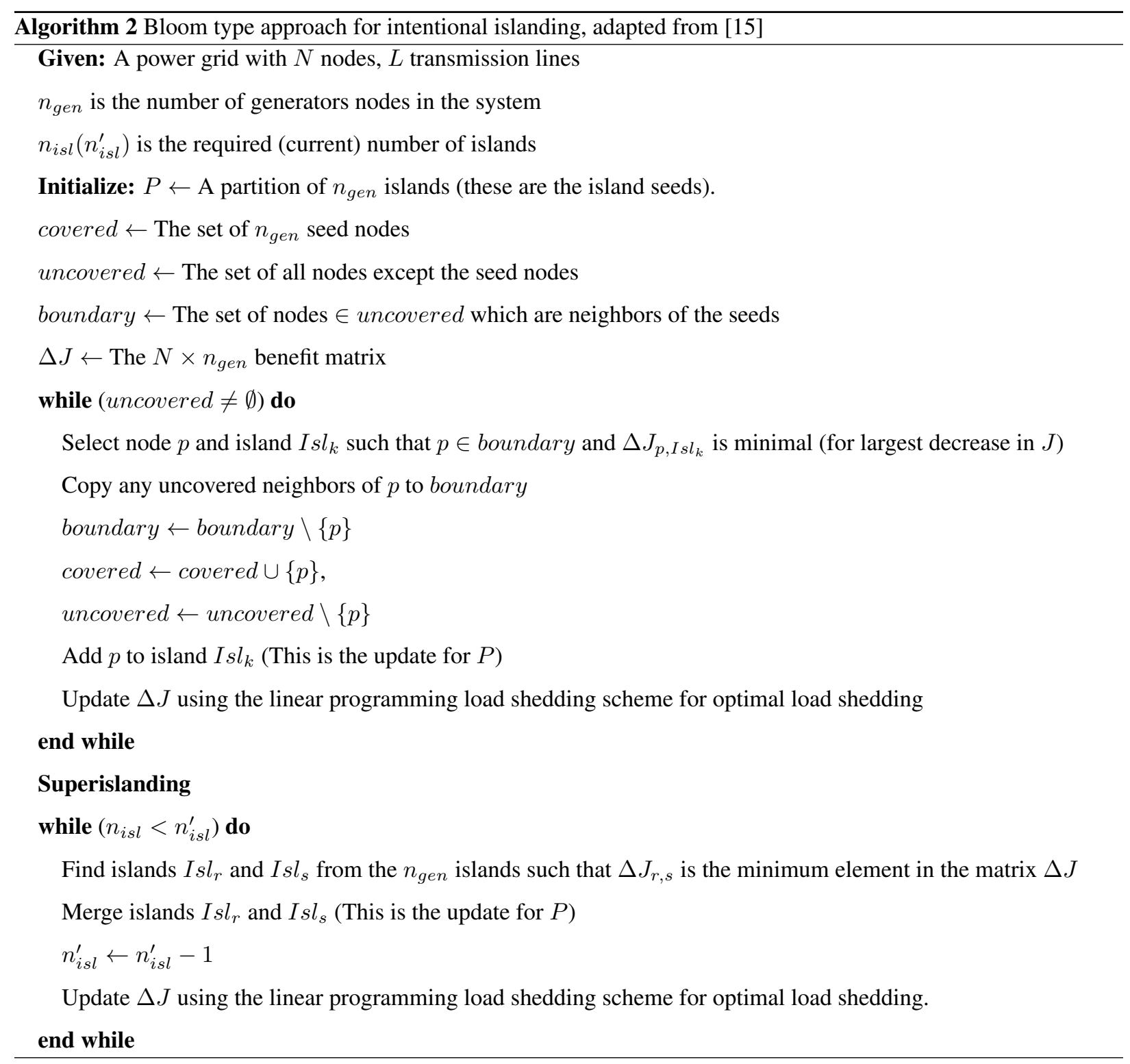




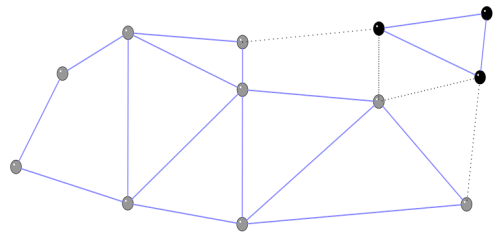

(a) Island 1

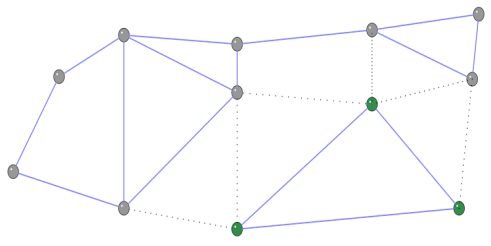

(b) Island 2

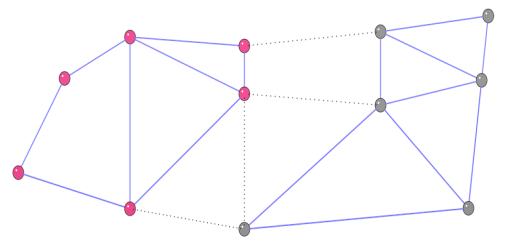

(c) Island 3

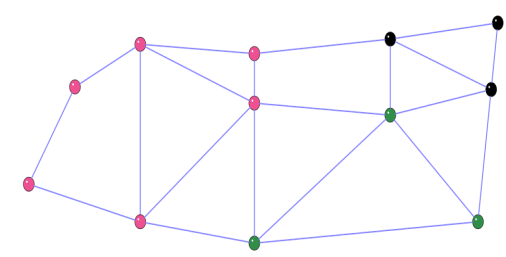

(d) Combined island structure

Figure C.1: The structure of three islands that belong to an example power grid with 12 nodes and 21 lines. Sub-figures 1(a)-1(c) show individual islands in different colors. These islands are connected with their topological complements by the dotted lines. Sub-figure 1(d) shows the combined island structure with each island represented by the corresponding color of the islands shown in sub-figures 1(a)-1(c) at the top. Color image available online. 


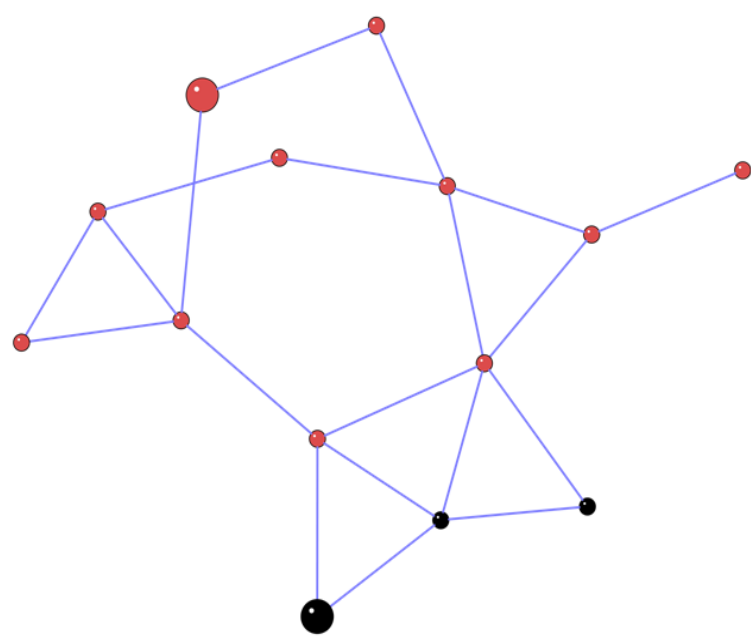

Figure C.2: Optimal islands for the 14 node system. The two islands are represented in a separate color and all nodes having the same color belong to the same island. The big circles represent the generators and small circles represent the load nodes. Figure available in color online. 


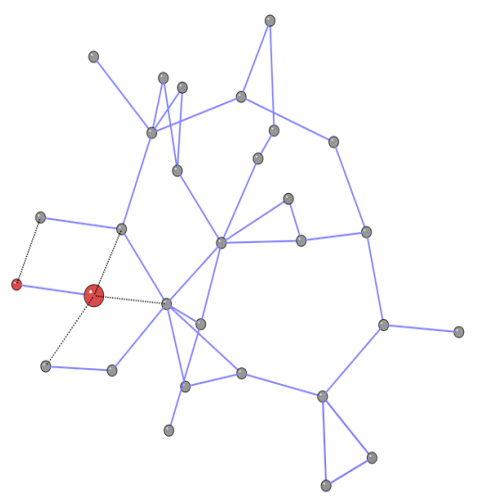

(a) Island 1

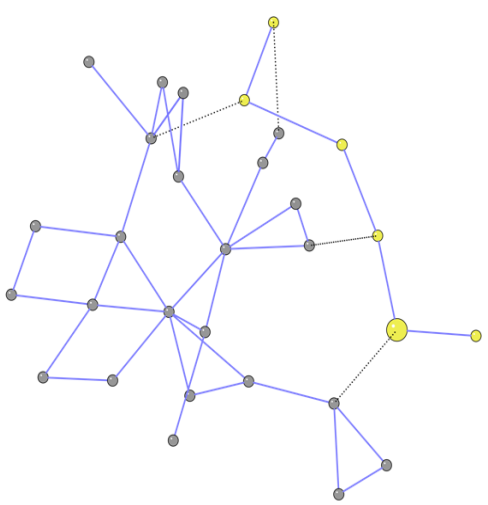

(d) Island 4

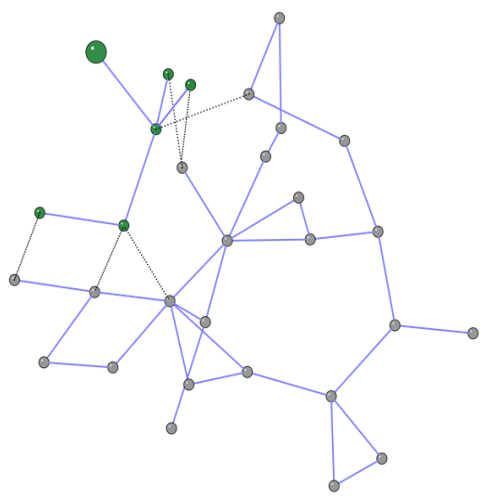

(b) Island 2

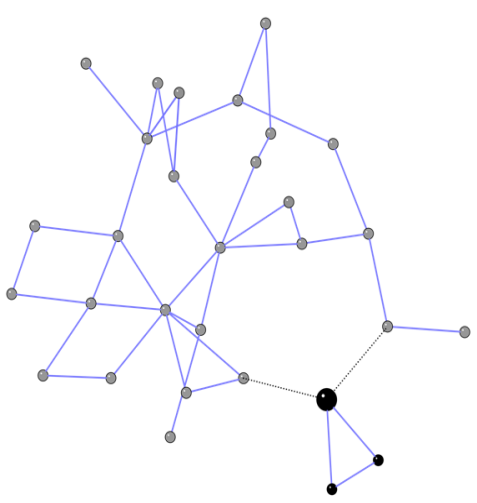

(e) Island 5

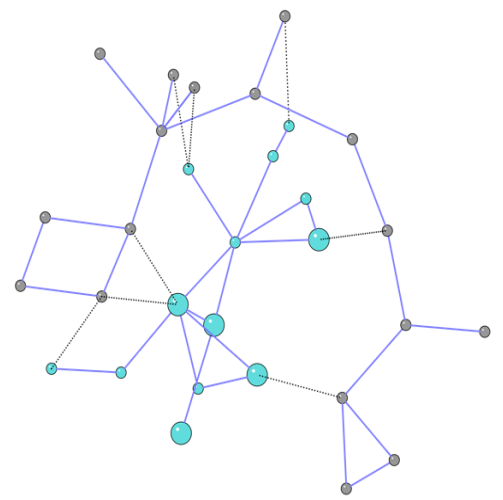

(c) Island 3

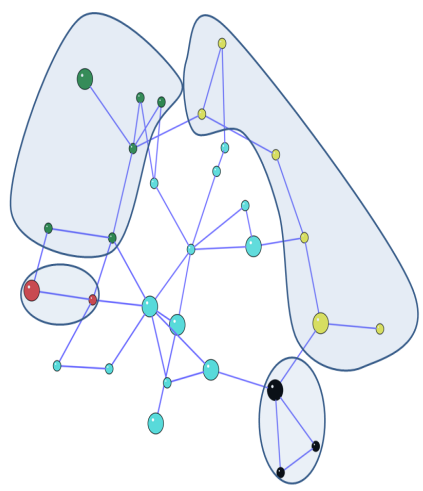

(f) Combined optimal island structure

Figure C.3: The optimal islanding solution for the 30-node system. The system has 9 generators and 21 load nodes. Every island is represented by a separate color and all nodes having the same color belong to the same island. The big circles represent the generators and small circles represent the load nodes. The sub-figures 3(a)-3(e) represent the 5 individual islands and the sub-figure 3(f) represents the combined optimal islanding solution. The generators are only shown in the respective island in sub-figures 3(a)-3(e). Figure available in color online. 


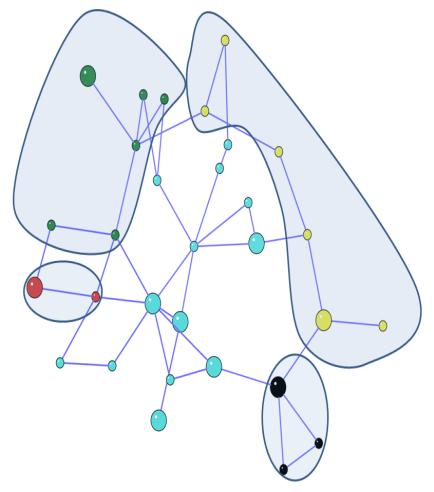

(a) Optimal

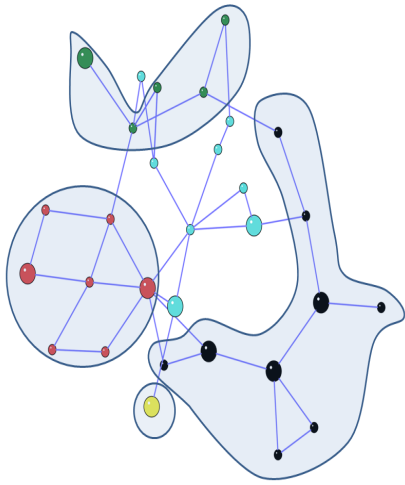

(b) Bloom type

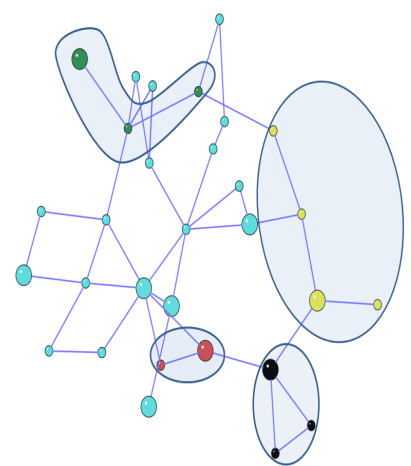

(c) Fast Greedy type

Figure C.4: The 5-island scenario for the 30-node system using optimization (a), Bloom type approach (b) and the Fast Greedy type approach (c). The difference in the island structure from these three approaches leads to different amounts of load shedding in the system. Figure available in color online.

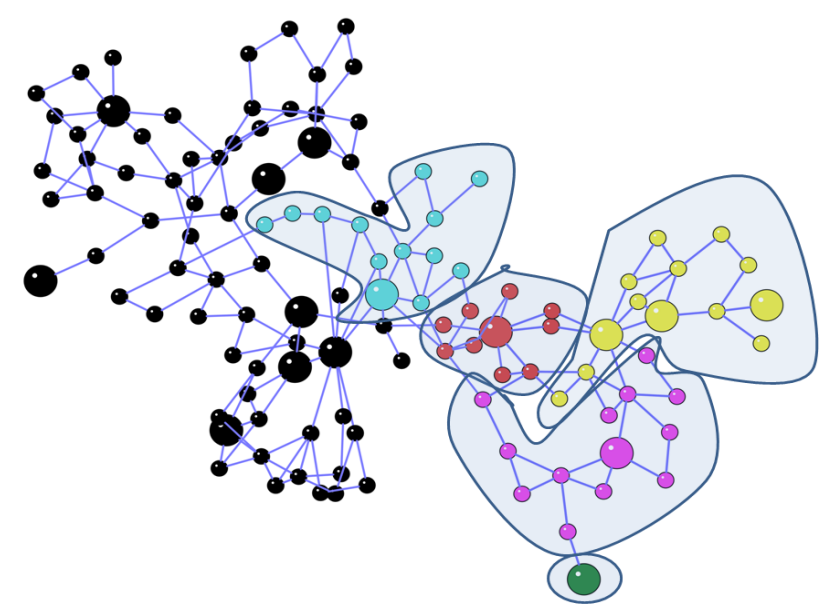

Figure C.5: The 6-island scenario for the 118-node system using Bloom type approach. Every island is represented by a separate color and all nodes having the same color belong to the same island. The big circles represent the generators and small circles represent the load nodes. Figure available in color online. 


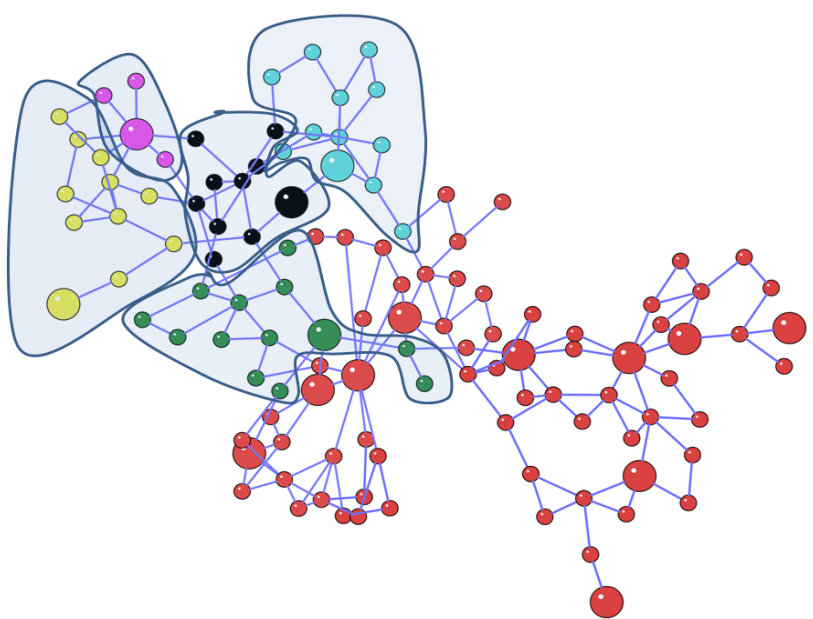

Figure C.6: The 6-island scenario for the 118-node system using Fast Greedy type approach. Every island is represented by a separate color and all nodes having the same color belong to the same island. The big circles represent the generators and small circles represent the load nodes. Figure available in color online. 
Table C.1: Definition of data inputs and decision variables

\begin{tabular}{|c|c|}
\hline Data input & Definition \\
\hline$a_{i, j}$ & $\begin{array}{l}\text { Binary adjacency matrix entry, } a_{i, j}=1 \text { if there is } \\
\text { a transmission line between nodes } i \text { and } j\end{array}$ \\
\hline$b_{i, j}$ & Admittance matrix entry of the transmission lines \\
\hline power $_{i}$ & Normal power/load at node $i$ \\
\hline$\alpha$ & Coefficient of minimum delivered load \\
\hline$c_{i, j}$ & Capacity of transmission line $(i, j)$ \\
\hline$n_{i s l}$ & Total number of islands \\
\hline$n_{\text {gen }}$ & Total number of generator nodes \\
\hline$n_{\text {loads }}$ & Total number of load nodes \\
\hline Decision variables & Definition \\
\hline$\mu_{i, j}^{k, s=1}$ & Binary variable equals 1 if line $(i, j)$ belongs to island $k$ \\
\hline$\mu_{i, j}^{k, s=2}$ & $\begin{array}{l}\text { Binary variable equals } 1 \text { if } 1) \text { line }(i, j) \text { belongs to } \\
\text { the topological complement of island } k \text {, and } 2) \text { line }(i, j) \text { is not } \\
\text { an interconnecting line between two islands }\end{array}$ \\
\hline$d_{i}^{k}$ & $\begin{array}{l}\text { Load decision variable at node } i \text {, which belongs to island } k \\
\text { or its topological complement. The variable } d_{i}^{k} \text { is negative } \\
\text { if the node } i \text { represents a generator, while it has a positive } \\
\text { value for load nodes. }\end{array}$ \\
\hline$\delta_{i}^{k}$ & $\begin{array}{l}\text { Voltage angle variable at node } i \text {,which belongs to island } k \\
\text { or its topological complement }\end{array}$ \\
\hline
\end{tabular}

Table C.2: Average percentage per island of total load remaining in the 14-node system by optimization and the two modularity approaches for 2 islands

\begin{tabular}{|l|lcll|}
\hline \multicolumn{4}{|c|}{ Average percentage of total load remaining } \\
\hline$n_{\text {isl }}$ & Optimal & Bloom type & $\begin{array}{l}\text { Fast } \\
\text { type }\end{array}$ & Greedy \\
\hline 2 & 86.28 & 86.28 & 86.28 \\
\hline
\end{tabular}


Table C.3: Average percentage per island of total load remaining in the 30-node system by optimization and the two modularity approaches for 5 islands

\begin{tabular}{|l|lcll|}
\hline \multicolumn{4}{|c|}{ Average percentage per island of total load remaining } \\
\hline$n_{i s l}$ & Optimal & Bloom type & Fast & Greedy \\
& & & type \\
\hline 5 & 93.84 & 60.65 & 59.94 \\
\hline
\end{tabular}

Table C.4: Average percentage per island of total load remaining in the 57-node system by the two modularity approaches for 2 islands

\begin{tabular}{|l|ll|}
\hline \multicolumn{3}{|c|}{ Average percentage per island of total load remaining } \\
\hline$n_{i s l}$ & Bloom type & Fast Greedy type \\
\hline 2 & 95.42 & 95.91 \\
\hline
\end{tabular}

Table C.5: Average percentage per island of total load remaining in the 118-node system by the two modularity approaches for different number of islands

\begin{tabular}{|l|ll|}
\hline \multicolumn{3}{|c|}{ Average percentage per island of total load remaining } \\
\hline$n_{\text {isl }}$ & Bloom type & Fast Greedy type \\
\hline 2 & 83.98 & 77.52 \\
\hline 3 & 86.36 & 77.43 \\
\hline 4 & 84.81 & 78.31 \\
\hline 5 & 83.76 & 78.76 \\
\hline 6 & 83.03 & 78.81 \\
\hline
\end{tabular}

Table C.6: Average percentage per island of total load remaining in the 247-node system by the two modularity approachesfor different number of islands

\begin{tabular}{|l|ll|}
\hline \multicolumn{3}{|c|}{ Average percentage per island of total load remaining } \\
\hline$n_{i s l}$ & Bloom type & Fast Greedy type \\
\hline 2 & 68.44 & 55.74 \\
\hline 3 & 63.36 & 43.87 \\
\hline 4 & 59.89 & 56.93 \\
\hline 5 & 57.92 & 59.56 \\
\hline 6 & 56.65 & 59.33 \\
\hline
\end{tabular}


[1] S. Mehdudia, and S. K. Ramachandaran, Worst outage cripples north India, The Hindu, July 2012 [Online].

Available: http: / /www.thehindu.com/news/national/article3702075. ece?homepage=true

[2] The Chosunilbo, Freak blackouts plunge Korea into darkness, September 2011 [Online].

Available: http://english.chosun.com/site/data/html_dir/2011/09/16/2011091600558.html

[3] J. Watson, Power failure leaves 5 million in the dark, San Fransisco Chronicle, September 2011 [Online].

Available: http://www.sfgate.com/news/article/Power-failure-leaves-5-million-in-the-dark-2310814. php

[4] North American Electric Reliability Corporation, Evaluation of Criteria, Methods, and Practices used for System Design, Planning, and Analysis Response to NERC Blackout Recommendation 13c [Online].

Available: http://www. nerc.com/docs/pc/tis/, 2005

[5] P. Hines, and S. Talukdar, Reciprocally altruistic agents for the mitigation of cascading failures in electrical power networks, Proceedings of the International Conference on Infrastructure Systems, Rotterdam, Netherlands, 2008.

[6] B. A. Carreras, V. E. Lynch, I. Dobson and D. E. Newman, Critical points and transitions in an electric power transmission model for cascading failure blackouts, CHAOS 12(2002), 985-994.

[7] S. Pahwa, C. Scoglio, and N. Schulz, Topological analysis and mitigation strategies for cascading failures in power grid networks [Online], Available: http://arxiv.org/pdf/1212.5620.pdf, 2012.

[8] D. Xu and A. A. Girgis, Optimal load shedding strategy in power systems with distributed generation, Proceedings of the IEEE PES. Columbus, OH, USA, January 2001.

[9] E. E. Aponte and J. K. Nelson, Time optimal load shedding for distributed power systems, IEEE T Power Syst 21(2006), 269-277.

[10] H. Bevrani, A. G. Tikdari and T. Hiyama, An intelligent based power system load shedding design using voltage and frequency information, Proceedings of the 2010 International Conference on Modeling, Identification and Control. Okayama, Japan, July 2010.

[11] J. Chen, J.S Thorp and I. Dobson, Cascading Dynamics and Mitigation Assessment in Power System Disturbances via a Hidden Failure Model, Int J Elec Power 27(2005), 318-326.

[12] IBM ILOG CPLEX Optimizer,

Available: http://www-01.ibm.com/software/integration/optimization/cplex-optimization-studio/

[13] PSTCA: Power Systems Test Case Archive [Online],

Available: http: //www.ee.washington.edu/research/pstca/

[14] M. E. J. Newman, Fast algorithm for detecting community structure in networks, Phys Rev E 69. 066133, 2004.

[15] P. Schumm, and C. Scoglio, Bloom: A stochastic growth-based fast method of community detection, JoCS 3. 356-366, 2012.

[16] A. Clauset, M. E. J. Newman, and C. Moore, Finding community structure in very large networks, Phys Rev E, 70. $066111,2004$.

[17] M. E. J. Newman, Modularity and community structure in networks, PNAS USA, 103. 8577-8582, 2006.

[18] S. Fortunato, Community detection in graphs, Phys Rep, 486. 75-174, 2010.

[19] M. E. J. Newman, and M. Girvan, Finding and evaluating community structure in networks, Phys Rev E, 69. $026113,2004$.

[20] S. Koch, S. Chatzivasileiadis, M. Vrakopoulou, and G. Andersson, Mitigation of cascading failures by real-time controlled islanding and graceful load shedding, 2010 IREP Symposium - Bulk Power System Dynamics and Control - VIII (IREP), 2010.

[21] M. El-werfelli, J. Brooks, and R. Dunn, Controlled islanding scheme for power systems, Proceedings of the 43rd International Universities Power Engineering Conference, 2008.

[22] H. You, V. Vittal, and X. Wang, Self-healing in power systems: an approach using islanding and rate of frequency decline-based load shedding, IEEE T Power Syst, 18. 174-181, 2003.

[23] X. Wang, and V. Vittal, System islanding using minimal cutsets with minimum net flow, Proceedings of the IEEE PES Power Systems Conference and Exposition, 2004.

[24] H. You, V. Vittal, and X. Wang, Slow coherency-based islanding, IEEE T Power Syst 19. 183-491, 2004

[25] B. Yang, V. Vittal, and G. T. Heydt, Slow Coherency-based controlled islanding - A Demonstration of the approach on the August 14, 2003 
blackout scenario, IEEE T Power Syst 21. 1840-1847, 2006.

[26] Z. Zhou, X. Bai, S. Zhao, Z. Li, J. Xu, X. Li, and W. Zhao, A new islanding boundary searching approach based on slow coherency and graph theoretic, Proceedings of the Fourth International Conference on Natural Computation, 2008.

[27] Q. Zhao, K. Sun, D. Zheng, J. Ma, and Q. Lu, A study of system splitting strategies for island operation of power system: a two-phase method based on OBDDs, IEEE T Power Syst, 18. 1556, 2003.

[28] K. Sun, D. Zheng, and Q. Lu, Splitting strategies for islanding operation of large-scale power systems using OBDD-based methods, IEEE T Power Syst, 18. 912-923, 2003.

[29] N. Fan, D. Izraelevitz, F. Pan, P.M. Pardalos, and J. Wang, A mixed integer programming approach for optimal power grid islanding, Energy Syst 3. 77-93, 2012.

[30] P. A. Trodden, W. A. Bukhsh, A. Grothey, and K. I. M. McKinnon, MILP formulation for islanding of power networks, IEEE T Power Syst: In Print.

[31] I. A. Hamad, B. Israels, P. A. Rikvold, and S. V. Poroseva, Spectral matrix methods for partitioning power grids: Applications to the Italian and Floridian high-voltage networks, Phys Procedia, 4. 125-129, 2010.

[32] I. A. Hamad, P. A. Rikvold and S. V. Poroseva, Floridian high-voltage power-grid network partitioning and cluster optimization using simulated annealing, Phys Procedia, 15. 2-6, 2011.

[33] R. Moreno, and A. Torres, Security of the power system based on the separation into islands, Proceedings of the IEEE PES Conference on Innovative Smart Grid Technologies, October 2011.

[34] W. Liu, L. Liu, D. A. Cartes, and G. K. Venayagamoorthy, Binary particle swarm optimization based defensive islanding of large scale power systems, Int J Comput Sci Applications, 4. 69-83, 2007.

[35] B. R. Gungor, Power Systems, Technology Publications, 1988.

[36] R. K. Ahuja, T. L. Magnati, and J. B. Orlin, Network Flows: Theory, Algorithms and Application, Prentice Hall, 1993. 